\title{
Féeries
}

Études sur le conte merveilleux, XVII $-\mathrm{XIX}{ }^{\mathrm{e}}$ siècle

$16 \mid 2020$

Le conte, les mythes antiques, la Bible

\section{Anne Defrance et Aurélia Gaillard (éds), Contes et Nouveau Recueil de contes de fées}

Paris, Honoré Champion, Série "Bibliothèque des Génies et des Fées ",

t. 11, 2 vol., coll. « Sources classiques », 2018, 1206 p.

\section{Emmanuelle Sempère}

\section{(2) OpenEdition}

Journals

Édition électronique

URL : https://journals.openedition.org/feeries/2967

DOI : $10.4000 /$ feeries. 2967

ISSN : 1957-7753

Éditeur

UGA Éditions/Université Grenoble Alpes

Édition imprimée

ISBN : 978-2-37747-194-2

ISSN : 1766-2842

Référence électronique

Emmanuelle Sempère, «Anne Defrance et Aurélia Gaillard (éds), Contes et Nouveau Recueil de contes de fées », Féeries [En ligne], 16 | 2020, mis en ligne le 01 décembre 2020, consulté le 10 décembre 2021. URL : http://journals.openedition.org/feeries/2967 ; DOI : https://doi.org/10.4000/feeries.2967

Ce document a été généré automatiquement le 10 décembre 2021.

(c) Féeries 


\section{Anne Defrance et Aurélia Gaillard (éds), Contes et Nouveau Recueil de contes de fées}

Paris, Honoré Champion, Série "Bibliothèque des Génies et des Fées ", t. 11, 2 vol., coll. « Sources classiques », 2018, 1206 p.

\section{Emmanuelle Sempère}

\section{RÉFÉRENCE}

Moncrif, Pajon, Contes et Anonyme, Nouveau Recueil de contes de fées, édités par Anne Defrance. Saint-Hyacinthe, Coypel, Godard de Beauchamps, Contes, édités par Aurélia Gaillard, Paris, Honoré Champion, Série « Bibliothèque des Génies et des Fées », t. 11, 2 vol., coll. « Sources classiques », 2018, 1206 p.

1 Cet ouvrage en deux volumes clôt la série de la "Bibliothèque des Génies et des Fées » dirigée par Nadine Jasmin et inaugurée en 2004 : riche de seize volumes désormais, cette collection témoigne de la diversité du genre féerique mais aussi de son unité profonde, renforcée par l'étroit dialogue qu'entretiennent les textes entre eux. Ce dernier tome, servi par une riche annotation qui tisse des liens avec les autres contes de la collection, illustre ces deux traits de façon exemplaire.

2 Les contes rassemblés dans ces deux volumes constituent en même temps un sousensemble bien identifié : les «conteurs rococo». Si la périodisation de ces textes couvrant un tiers de siècle, de 1715 (Moncrif, "Zéloïde ») à 1747, peut sembler large, elle correspond précisément à ce que les historiens d'art et de littérature identifient comme la période rococo, dont l'un des sommets est le Salon de 1746 commenté par La Font de Saint-Yenne. On perçoit d'emblée que l'enjeu de cette édition déborde le seul champ du conte en tant que tel : en offrant aux lecteurs, parfois pour la première fois depuis le $\mathrm{XVIII}^{\mathrm{e}}$ siècle, des textes précisément établis et annotés, ces deux volumes permettent de se faire une idée de ce que pourrait désigner un nom de genre qui serait 
le «conte rococo». Des traits frappent d'emblée, au travers de la variété de leur réalisation: le champ sémantique et symbolique de la vue, empêchée, augmentée, déformée, réfractée ou simplement médiatisée (les miroirs surtout sont omniprésents), celui du change et de la mutabilité (les métamorphoses merveilleuses exprimant l'inconstance des êtres autant que la variation de leur apparence), le goût du plaisir et la crainte de sa disparition, un rapport au temps où priment l'immédiateté et l'oubli, etc.

3 L'introduction générale met en avant ce que le conte rococo doit à l'esthétique rococo. En premier lieu « l'art des glaces et des simulacres » (p. 11), qui pousse l'enchantement à son "comble» tout en le mettant à distance: le merveilleux "plie la réalité à l'illusion, fait de l'illusion la seule réalité » (p.12). Un second trait découle du premier, c'est la « réflexivité du conte » (p. 12). Objet de nombreuses notes au fil des textes, cette réflexivité se précise en une "forme d'incrustation métacritique»: les contes véhiculent et mobilisent la vaste " mémoire orale des contes » (p.14) mais aussi celle de toute la production mondaine des contes depuis Perrault et Aulnoy. Le conte rococo affiche ainsi «la présence d'une voix de conteur» (p.15) pour mieux affirmer une intention morale qui n'est jamais bien loin quoique rarement totalement sérieuse. Le troisième trait mis en avant par cette remarquable introduction est " l'intergénéricité " de ces textes qui entremêlent les emprunts jusqu'à «l'estompe » (p. 15) : il ne s'agit pas tant de parodier le merveilleux - et il l'est, assurément - que de réaliser "l'épanchement [du conte] dans le romanesque» (p.16). Dérision et morale s'en trouvent alors paradoxalement conjointement favorisées. Le dernier trait est peut-être formulé de façon un peu restrictive : par cette "métaphysique de l'amour » - celle de Marivaux - c'est toute la question de «l'inconstance» de l'être qui est concernée, laquelle n'est pas qu'amoureuse mais questionne aussi l'identité personnelle.

Ces traits clairement définis ne dessinent pour autant aucun canevas fixe, comme le soulignent les deux éditrices à la fin de cette introduction : rassembler ces vingt-quatre " contes rococo » met au contraire en évidence à la fois ce qui les unit et leur richesse propre, où chaque lecteur aura sans doute ses coups de cœur, découvrant l'inventivité parfois bizarre de Pajon ou de Godard de Beauchamps, la complexité des systèmes politico-moraux de Saint-Hyacinthe, la finesse de Moncrif, les expérimentations esthétiques de l'anonyme et de Coypel.

Entrons dans le détail de ce beau volume. Le premier tome rassemble trois ensembles édités par Anne Defrance: les contes de Moncrif, Le Nouveau Recueil de contes de fées, anonyme, et les contes de Pajon, sur une période allant de 1715 à 1745.

6 Les quatre contes de Moncrif donnent à voir « une sorte de kaléidoscope : la morale éclairante qui les sous-tend recompose en permanence images et figures pour servir le propos " (Introduction, p. 27) et le lecteur appréciera l'inventivité formelle, mais aussi la connaissance du cœur, qu'y manifeste Moncrif. Les Aventures de Zéloïde et d'Amanzarifdine (1715), qui deviennent l'année suivante Les Mille et Une Faveurs, constituent un véritable " petit roman de mœurs » (Notice, p. 49) dont l'objet pourrait être les inconvénients de la sincérité, anticipant les questionnements de Marivaux dans Le Monde vrai du Cabinet du philosophe (1734) et invitant les lecteurs et les lectrices à réconcilier le plaisir et la morale avec une grande liberté et surtout, dans une nouvelle égalité entre les sexes. L'ambition morale s'affirme plus encore dans les cinq contes de 1738, étroitement associés aux Essais sur la nécessité et les moyens de plaire dont ils constituent formellement la seconde partie et, pour le fond, la mise en pratique et la 
réélaboration expérimentale bien plus que de simples illustrations. La « dynamique de lecture» (Notice, p.139) induite par ce dispositif textuel est remarquablement analysée, au travers, entre autres, d'un parallèle avec Fénelon, le «modèle " avoué de Moncrif qui en adopte la démarche "démystificatrice" envers le merveilleux. Ce parallèle met en évidence l'innovation de Moncrif sur le terrain de l'expérimentation, "[c]hacun des cinq contes constitu[ant] un laboratoire expérimental dont les personnages imaginaires sont les cobayes » (p. 137). Moncrif apparaît ainsi comme un moraliste singulier, assez libre d'esprit pour questionner les fondements de l'ordre politique ("Les Dons des fées», "Alidor et Thersandre») et rechercher "le vrai mérite» (Notice des «Aïeux», p. 166): auteur roturier à l'« ascension sociale fulgurante " (Introduction, p. 26), Moncrif peint un monde où «la mort sociale est devenue le vrai tragique de l'individu» (Notice des "Voyageuses», p. 183). Sauf à réinventer les conditions de sa propre existence: c'est l'un des propos de son conte sans doute le plus, sinon le mieux connu, Les Âmes rivales (1738), dont l'édition présente judicieusement les deux versions (le premier état du texte de 1732 est donné en annexe), permettant d'apprécier comment Moncrif avance dans l'exploitation fictionnelle d'un motif emprunté à la critique religieuse. La Notice fait le lien entre les données historiques concernant le motif de la métempsycose, diffusé par les missionnaires jésuites, et les enjeux philosophiques et littéraires de ses multiples applications dans la littérature contemporaine. "Affabulant sur ce qui n'était déjà, à l'origine, rien d'autre qu'une affabulation» (Notice, p. 200), Moncrif fait de la métempsycose un propulseur d'invention. Le propos est aussi, à nouveau, « une étude des caractères et des mœurs " au point de "sign[er] la mutation contemporaine du conte oriental en conte moral» (Notice, p. 205). Mais Moncrif renoue aussi dans ce conte avec l'inspiration libertine des Mille et Une Faveurs, profitant de ce que l'interaction amoureuse est démultipliée par la migration des âmes dans les corps de l'amant, du rival et de la maîtresse : «les fantasmes affleurent » (Notice, p. 202), sans rien sacrifier à «l'humour » puissant de Moncrif. Un autre fil continu de l'œuvre de Moncrif, et qui se retrouve dans de nombreux contes de la période, est très justement mis en valeur par Anne Defrance : il s'agit de la dynamique d'invention induite par la proximité avec l'opéra, qu'il s'agisse d'insertion de morceaux chantés (par exemple dans Les Aventures de Zéloïde et d'Amanzarifdine, p. 101-103) ou de la transposition du conte en livret, comme ce fut le cas pour Les Âmes rivales, dont Moncrif rédigea luimême une version opératique reproduite en annexe (p. 240-245). En quelques pages le lecteur pourra retrouver comme une épure de la fable de l'inconstance que constitue l'image de la transmigration : c'est en quelque sorte « la folie expliquée par elle-même » («Les Âmes réunies, ou La Métempsycose », p. 241).

7 Anonyme, Le Nouveau Recueil de contes de fées (1731, la première édition de 1718 n'ayant jamais été retrouvée), est emblématique du mode de production d'un grand nombre de contes de la période : la réécriture, l'écriture collective et en définitive l'anonymat produisent un «tissage des motifs merveilleux» (Notice du «Navire volant», p. 315) entrelacés de traits galants et moraux. Sa parfaite connaissance des contes de la période permet à l'éditrice de mettre au jour tel ou tel emprunt, à Mailly, à Murat, à Aulnoy, ou encore à Basile et à « la littérature internationale elle-même passée dans le folklore" (Introduction, p. 267). Plusieurs erreurs d'attribution ou d'identification de sources sont corrigées, avec une grande précision (c'est en particulier le cas d'« Alphinge », Notice, p. 384-385) : l'interprétation des textes s'en trouve grandement enrichie autant que la connaissance des chaînes de transmission de conte à conte. Le 
Nouveau Recueil frappe par son inventivité colorée et son jeu sur les règnes, jouant des métamorphoses animales, des objets hybrides et des êtres mi-animés et mi-inanimés, tels ce "petit char de girasol dont les roues étaient des topazes", "tiré par six vers luisants » («Le Prince Périnet », p. 340) ou ces poupées de plâtre miniaturisées, dont le lecteur découvre la "beauté [...] très éclatante et très solide " ("Le Navire volant ", p. 323). À ces jeux de matières, de couleurs et de formes emblématiques du rococo s'ajoutent de nombreux motifs empruntés aux arts décoratifs, à l'architecture ou à l'art des jardins de la période, tel ce «berceau de jasmin et de chèvrefeuilles » où les paroles des amants, quoique dites "à voix basse », s'entendent "très distinctement " ("Le Buisson d'épines fleuries », p. 373). Les sensations auditives, tactiles et visuelles sont perpétuellement exacerbées par de tels dispositifs, dont l'ingéniosité merveilleuse renforce les effets. C'est peut-être dans le dernier conte que se fait le mieux sentir l'« effet de vérité » (Introduction, p. 273) produit par la présence de la voix du conteur anonyme et qui est, parfois, sa seule intervention sur les contes sources (ainsi pour «Les Perroquets », voir les notes du conte, p. 307-314). Dans « Le Médecin de satin » en effet, dont Anne Defrance montre la parenté, plus lointaine certes, avec "L'Oiseau bleu » d'Aulnoy, la voix auctoriale fait résonner la « voix charmante » des personnages (p. 424) pour mieux glorifier « l'amour vertueux » (Notice, p. 411).

8 C'est un univers autrement noir que celui des contes de Pajon, mais pour une large part aussi mal connu. Composés dans le cadre mondain des sociétés littéraires du Paris des années 1740, en particulier celle du Bout-du-banc à laquelle appartenait aussi Moncrif (Introduction, p. 458) les contes de Pajon usent du suspens et de la relative indifférence de la fin, renversant en loi du genre la critique d'Horace envers les mauvaises fins: Desinit in piscem mulier formosa superne (" Un beau buste de femme qui finit en poisson ", Introduction p. 457 et Annexe p.685). Anne Defrance précise en effet que c'est probablement Pajon lui-même qui reprend ce vers dans un article du Mercure pour caractériser ses propres contes et en faire les parangons d'une esthétique déceptive: " Désormais quand les contes qu'on vous enverra n'auront que la première moitié de bonne, contentez-vous de promettre la deuxième et ne tenez jamais parole» (p. 685). Les quatre contes proposés se laissent scinder en deux inspirations, l'une plutôt orientale, l'autre galante pour l'Histoire du roi Splendide et de la Princesse Hétéroclite ». La parodie domine, ainsi que l'irrévérence, ce qui n'empêche pas un propos moral appuyé de maximes. On lira ainsi dans Les Enchanteurs ou La Bague de puissance : "Souvenez-vous que la puissance doit être accompagnée de la justice, et que la justice exige une exacte connaissance de la vérité » (p. 522). La fin du Manuscrit tiré de l'arabe fait aussi bonne place à l'utopie politique, liant le "bonheur [des] peuples " à la "félicité pure » des «époux fortunés » qui les gouvernent et qui « auraient voulu les rendre aussi heureux qu'ils l'étaient eux-mêmes» (p. 501). Mais c'est par les motifs tirés de l'astrologie et de la cabale, qu'il ramène vers « la sphère fantastique » (Notice du Manuscrit, p. 476), et par la récurrence d'épreuves qui ne reculent pas devant les effets d'horreur que Pajon se montre le plus original : ainsi de ces têtes coupées et transplantées dans l'Histoire des trois fils d'Hali Bassa de la mer et des filles de Siroco, qui font écho à la transmigration des "âmes rivales» de Moncrif mais anticipent aussi la noirceur de l'épisode si connu de l'ollivier de Cazotte qui fit rêver Nerval. L'humour noir désamorce l'effroi lorsque les deux amants aux têtes échangées peuvent se féliciter d'être " par un bonheur inouï, [...] condamnés à avoir [à nouveau] la tête tranchée » (p. 589). Quant aux mésaventures d'une marmite "immobile d'étonnement" puis bien embarrassée de traverser "un potager» sur ses «trois pieds», elles font d'autant plus sourire que le jeu 
métamorphique bouscule la métaphysique des cœurs (car Marivaux à nouveau n'est pas loin, voir p. 566 et la Notice p. 545). Mais les traits d'invention de Pajon échappent au relevé. Citons seulement pour finir le reflet de deux visages dans un même miroir, qui résonne si bien avec la scène 7 de La Dispute de Marivaux: "Mais regardez dans le miroir, votre visage est tout près du mien, l'un n'efface pas l'autre» (p. 679), et chez Marivaux, "Nos visages vont se toucher ...] à présent je ne vois plus que moi » ( $L a$ Dispute, éd. J. Goldzink, Paris, GF-Flammarion, 2017, p. 167).

9 Le second tome rassemble, édités par Aurélia Gaillard, les contes de Saint-Hyacinthe (près de 280 pages), de Godard de Beauchamps (30 pages) et de Coypel (15 pages). S'ils diffèrent nettement par la taille, ces contes partagent l'art du "pot-pourri » (Notice de Funestine, p. 1017) et du «métatexte " (Histoire du Prince Titi, p. 890, commentaire en note). Le court conte de Coypel lui-même apparaît comme " un conte des contes " (note d'« Aglaé ou Nabotine ", p. 1123). Leur période de composition est également assez homogène (1736 pour l'Histoire du Prince Titi, 1737 pour « Funestine », entre 1735 et 1752 pour Aglaé ou Nabotine). Aurélia Gaillard montre ici comment la plasticité du genre véhicule nombre de motifs majeurs du rococo, de l'esthétique à l'imaginaire des objets et des règnes naturels, sans faire oublier, en particulier chez Saint-Hyacinthe, les préoccupations sociales et politiques qui transparaissent sous le voile de la légèreté. Tous trois renvoient aussi assez nettement aux décors et à l'esthétique du rococo, et si Coypel n'évoque pas directement les arts dans Aglaé, son œuvre essentiellement critique et esthétique fait largement l'arrière-plan du conte. Le trait esthétique est particulièrement exhibé dans Funestine, qui cite les figures "grotesques" et «bizarres » de «Calot» (p. 1047) et incarne le «Clair-Obscur» dans un personnage qui trace des « hiéroglyphes » qui sont autant d'anamorphoses (p. 1041). L'art du change, si présent chez Moncrif et tous les conteurs rococo, est au cœur du Prince Titi, où il s'exprime dans la sphère sentimentale sous la forme d'une inconstance heureuse (voir les lois du royaume de Félicie, p. 880-883), comme dans l'univers des objets (des fruits devenant diamants, p. 749). Mais « la clef de voûte » du conte et de « la pensée de SaintHyacinthe » est "l'attachement à la vertu » (note, p. 888), aussi bien dans la sphère privée que publique, où il n'y a pas jusqu'à la «frivolité [qui] ne puisse être une leçon politique» (note, p. 752). La démystification du pouvoir qu'opère le merveilleux en montre le grotesque. Ainsi du discours de l'inquiétante et autoritaire Tripalle : « Nous avons la justice pour nous, nous avons aussi la force, car nous avons l'argent » (p. 820). La modernité de la pensée politique se précise au fil de l'annotation de ce texte surprenant et souvent déroutant, qui emprunte à Montesquieu pour aussi s'en écarter et anticiper Rousseau, voire l'abbé Grégoire ou Condorcet (note, p. 843). Le conte rococo ne renonce pas loin s'en faut aux motifs merveilleux traditionnels, voire archaïques, comme celui de l'enfermement dans la tour (Le Prince Titi, p. 807, et voir également l'exemple de l'eau ou de la liqueur merveilleuse, p. 925), mais il les entrelace à d'autres et les reconfigure, par exemple ici à une dimension pseudo-historique et surtout politique. Les épreuves (le gobelet puis le sabot, p. 867), prennent ainsi une nouvelle dimension: "L'épreuve est alors la forme 'merveilleuse' que prend le thème de l'éducation du prince » écrit Aurélia Gaillard (note, p. 870). Il s'agit bien, dès lors, d'une « fable, une 'allégorie royale' [sous-titre du conte], qui n'éloigne de la réalité que pour permettre de la mieux penser » (Notice, p. 714) : réalité sociale, économique, politique, judiciaire, etc., en bref tous les sujets qui pouvaient intéresser « une pensée réformiste, parfois radicale » (Notice, p. 717). 
10 Avec Godard de Beauchamps, le lecteur peut se croire en terrain plus familier, celui du conte extravagant, dont la matière sinon la cible est le merveilleux lui-même : le conte le convie au grand «massacre des fées " (Notice de Funestine, p. 1028), qui entérine le " virage de la féerie vers une autre matière et surtout manière de narrer, mi-sérieuse mi-ironique, qui laisse une large part au pastiche et au recyclage » (p. 1029). Le conteur lui-même parle d'un "anéantissement " (p. 1104). Mais la lucidité ne semble induire nulle supériorité, car si «[c]eux qui ignorent [l'inexistence des fées], les mettent tous les jours en œuvre comme des êtres existants[,] ceux qui sont mieux instruits font de vaines tentatives pour les ressusciter, ils ne leur substituent que des avortons, que des éphémères » (p. 1104). Le merveilleux se situe ainsi dans un « clair-obscur » qui souvent tend au sombre, soit que la "féerie extravagante tir[e] vers le fantastique" (Introduction, p. 1017), soit que le merveilleux vire au tragique, comme avec les « songes funestes [de Funestine], dont l'horreur ne s'évanouissait point à son réveil » (p. 1055) et qui font clairement écho à Prévost : Aurélia Gaillard montre comment le conte rococo dépayse le regard en brouillant les frontières du merveilleux et de la réalité. On conviendra sans peine, dès lors, que le " pot-pourri de traditions et formes narratives, chroniques, nouvelle historique et sentimentale, conte galant, récit de voyage » qui caractérise Funestine (Introduction, p. 1017) mérite une véritable "réhabilitation» (Notice, p. 1025), d'autant que le " pastiche » et le « recyclage » s'y combinent avec « un projet 'philosophique' » sans cesse "stoppé » par « le jeu ambigu que mène le conteur entre dévoilement allégorique convenu, dénigrement et revendication du genre " (p. 1028-1029).

11 Le court conte de Coypel qui ferme cet ensemble pourrait à certains égards en apparaître comme une esquisse, une épure qui mettrait en valeur un trait parmi les plus intéressants de ces contes rococo, à savoir cette "métaphysique » du cœur qui « creuse la complexité des petites attitudes, petits gestes et des subtilités du sentiment de l'amour-propre notamment" (Notice d'Aglaé ou Nabotine, p. 1121). La brièveté du conte s'accompagne d'un art proprement saisissant de peindre les âmes en mouvement où se reconnaît « l'empreinte de Marivaux » (ibid.) mais qui doit aussi beaucoup à l'œil du théoricien de la peinture. Ainsi du trouble de Nabotine, qui n'est pas encore devenue Aglaé : «[...] l'embarras de Nabotine allant toujours en augmentant, suffisait pour la rendre plus silencieuse qu'à l'ordinaire, et le désir de surmonter cet embarras, acheva de lui ôter l'usage de la parole ; car il suffit d'avoir envie de bien dire, pour ne plus rien dire du tout. On rêve longtemps pour trouver quelque chose de joli : honteux d'avoir rêvé, on rêve de nouveau au moyen de réparer sa faute, et s'il arrive, par hasard, qu'on trouve, à force de rêver, quelque chose de bon ; on est étonné que ce quelque chose soit venu trop tard et que la conversation ait changé de matière pendant que l'on rêvait. " (p. 1129).

12 Complétés comme c'est d'usage dans la série, d'utiles résumés et index, mais aussi d'annexes particulièrement éclairantes et riches, ces «contes rococo " réservent, on l'aura compris, de très beaux moments de lecture, où se trouvent satisfaits aussi bien l'esprit que le cœur, le goût du merveilleux et celui de la philosophie, respectant le principe énoncé par Saint-Hyacinthe, qu'« un récit merveilleux attache toujours plus que l'exposition raisonnable de la vérité » (p. 891). Quant à l'appareil critique de cet ultime opus de la série de la "Bibliothèque des Génies et des Fées ", il faut en saluer la remarquable « exposition » qui parvient à faire oublier l'érudition de l'information et la profondeur de l'analyse par la souplesse du style. 


\section{AUTEURS}

\section{EMMANUELLE SEMPÈRE}

Université de Strasbourg, Configurations littéraires EA 1337 